\title{
Herramientas para el aprendizaje colaborativo en ciencias alimentarias y de la salud en la enseñanza universitaria y de formación profesional
}

\section{Tools for cooperative learning in food and health sciences in university education and vocational training}

\begin{abstract}
Fernando Cámara-Martos ${ }^{1 *}$, Rafael Moreno-Rojas ${ }^{2}$, Jesús Sevillano-Morales ${ }^{3}$, Alicia Moreno-Ortega ${ }^{4}$, Inmaculada Rodríguez-Delgado ${ }^{5}$, Manuel Ángel AmaroLópez $^{6}$, Rafael Gómez-Díaz ${ }^{7}$, Montserrat Vioque-Amor ${ }^{8}$, Francisca Ruíz-Sutil ${ }^{9}$, Dolores Salcedo-Jiménez ${ }^{10}$, Carmen Sánchez-Narváez ${ }^{11}$, María Luisa Ortíz de Andrés ${ }^{12}$ y María Carmen Dastis-Martín ${ }^{13}$
\end{abstract}

Fecha de recepción: 18/03/2019; Fecha de revisión: 09/06/2019; Fecha de aceptación: 19/07/2020

Cómo citar este artículo:

Cámara-Martos, F. et al. (2019). Herramientas para el aprendizaje colaborativo en ciencias alimentarias y de la salud en la enseñanza universitaria y de formación profesional. Revista de Innovación y Buenas Prácticas Docentes, 8(3), 122-129.

Autor de Correspondencia: fernando.camara@uco.es

\begin{abstract}
Resumen:
El presente proyecto ha pretendido la realización de prácticas docentes por parte de alumnos de $4^{\circ}$ curso del Grado de Ciencia y Tecnología de los Alimentos, basadas en conocimientos sobre alimentación y nutrición, en colaboración con los estudiantes de un Ciclo Formativo de Grado Superior de la Familia Profesional Sanitaria en el Instituto de Educación Secundaria Fuensanta de la ciudad de Córdoba. La toma de decisiones y realización de las mismas se ha llevado a cabo en todo momento por parte del alumnado de ambos centros educativos, actuando el profesorado como meros orientadores del proceso. Con dicho proyecto se ha pretendido, además de la puesta en práctica de los conocimientos teóricos, adquiridos durante su formación, la puesta en común de una misma actividad que permita el conocimiento entre ambos niveles educativos así como el enriquecimiento profesional y personal.
\end{abstract}

Palabras clave: antropometría, educación nutricional, encuestas alimentarias, prácticas educativas

\begin{abstract}
:
The object of the present project has been the realization of teaching practices by students of 4 th year of Degree in Food Science and Technology of the University of Cordoba in collaboration with vocational training students. They have made decision-making at all times and professors has been mere counselors. With this project it has been tried, in addition to the putting into practice of the theoretical knowledge, acquired during its formation. Moreover it has been keep in touch, students belonging to different educational stages
\end{abstract}

Key Words: anthropometry, educational practices, food surveys, nutritional education

\footnotetext{
1 Universidad de Córdoba (España), fernando.camara@uco.es; ORCID: 0000 - 0003 - 1171 - 1062

2 Universidad de Córdoba (España), rafael.moreno@uco.es; ORCID: 0000-0003-3134-7392

3 Universidad de Córdoba (España), jsevillano@uco.es; ORCID: 0000 - 0001 - 9617 - 5152
} 


\footnotetext{
${ }^{4}$ Universidad de Córdoba (España), t22moora@uco.es; ORCID: 0000 - 0002 - 2892 - 4294

${ }^{5}$ Universidad de Córdoba (España), v62rodem @uco.es; ORCID: 0000-0003-2417-3910

${ }^{6}$ Universidad de Córdoba (España), bt1amlom@uco.es; ORCID: 0000-0003-4845-6026

${ }^{7}$ Universidad de Córdoba (España), bt1godir@uco.es; ORCID: 0000-0003-4582-3193

8 Universidad de Córdoba (España), bt1viamm @uco.es; ORCID: 0000-0002-0860-3362

9-13 Profesorado del Instituto de Educación Secundaria Fuensanta. Avenida de Calderón de la Barca, s/n, 14010 Córdoba.
} 


\section{INTRODUCCIÓN}

Hasta hace bien poco el devenir de la Universidad y la Formación Profesional en España han transcurrido por caminos bien distintos, en el sentido que la enseñanza universitaria era la elección que escogían todos los alumnos que poseían una cierta capacidad para estudiar, mientras que aquellos estudiantes que no la poseían se veían abocados a otro tipo de estudios entre los que se encontraban los de Formación Profesional (FP).

Así, la FP era una rama del sistema educativo que surgió por la necesidad de regular las salidas profesionales de los estudiantes que no querían continuar estudiando una carrera universitaria y que únicamente buscaban una salida laboral. Surgió orientada y aplicada para muchas ramas profesionales, tanto sanitaria como química, y otras de carácter científico - tecnológico, como de la administración, turismo, actividades deportivas, etc. La FP se reguló por primera vez el 3 de octubre de 1990, por medio de la LOGSE, y fue modificada posteriormente por la LOE del 3 de mayo del 2006

En la actualidad todo esto ha sido modificado radicalmente. Los cambios en el mercado laboral, mediante los cuales es muy probable que un trabajador no trabajará toda su vida profesional en una misma ocupación y empresa, las necesidades de una actualización permanente de conocimientos y de formación continua, hacen que muchos estudiantes de FP, antes o después, continúen su periodo de formación con la realización de unos estudios universitarios e incluso que muchos titulados universitarios, ante una posible situación de desempleo, acaben complementando su titulación universitaria con unos estudios de FP relacionados con ella, con el objetivo de adquirir una enfoque más técnico o profesional en algunos casos.

Por todo ello, y considerando que la Universidad tiene en los estudiantes de FP una más que considerable cantera de posibles futuros estudiantes, creemos de primordial importancia la realización de actividades conjuntas entre estudios y titulaciones de un mismo ámbito o Familia Profesional (en términos de FP) que fomenten el conocimiento, la cooperación y el contacto entre estudiantes y profesorado de ambas etapas educativas. Al mismo tiempo, si la realización de estas actividades conjuntas son de carácter práctico, con interrelación de diferentes asignaturas y en las que se puedan aplicar a situaciones reales los conocimientos teóricos adquiridos creemos más que justificada la propuesta.

\section{OBJETIVOS}

El principal objetivo de este proyecto de investigación fue estudiar la posible relación existente entre un conocimiento en distintos tipos de materias como a) nutrición, b) tecnología alimentaria, c) seguridad alimentaria y d) gastronomía y el estado nutricional de grupos de estudiantes pertenecientes a los Ciclos Formativos del Instituto de Educación Secundaria Fuensanta (en adelante IES Fuensanta) de la ciudad de Córdoba. Este estudio fue realizado íntegramente por estudiantes de $4^{\circ}$ Curso del Grado de Ciencia y Tecnología de Alimentos de la Universidad de Córdoba y de $2^{\circ}$ Curso de Ciclo Formativo de Grado Superior (CFGS) de Dietética del IES Fuensanta. 
Todas las actividades fueron realizadas de manera autónoma por los alumnos de ambas titulaciones, actuando sus profesores únicamente como meros orientadores. Se ha pretendido que el alumnado tome de forma responsable y autónoma sus propias decisiones, con la obtención de resultados positivos en determinados casos o de tener que realizar medidas correctoras en caso de que el resultado obtenido fuera negativo. Introducimos así al alumno a exponerse a situaciones con las que tendrá que convivir de manera inminente en su trabajo profesional, cuando finalice sus estudios y acceda al mercado laboral.

Este principal objetivo, lleva a su vez aparejado otra serie de objetivos secundarios como:

- Promover la cooperación entre estudiantes y profesorado de centros docentes universitarios y no universitarios en el marco de actividades académicas de carácter práctico.

- Ofrecer a los alumnos capacidad de decidir el desarrollo de sus prácticas.

- Fomentar nuevas aplicaciones basadas en las TICS para el desarrollo de las prácticas.

Aplicar los conocimientos teóricos adquiridos en determinadas asignaturas del Grado de Ciencia y Tecnología de los Alimentos a situaciones reales para la valoración del estado de salud de una población.

\section{DESARROLLO DE LA EXPERIENCIA DE INNOVACIÓN}

El desarrollo del presente proyecto comenzó el primer día de clase de la asignatura Nutrición Aplicada del Grado de Ciencia y Tecnología de los Alimentos donde se informó a los alumnos pormenorizadamente de cada una de las tareas a realizar. A continuación, se nombraron unos alumnos que se responsabilizarían de la organización y realización de las distintas fases del proyecto. Estas fases de desarrollo del proyecto fueron: a) Búsqueda y recopilación bibliográfica, b) Elaboración de la ficha de recogida de datos antropométricos, c) Elaboración de las encuestas de conocimientos alimentarios (en sus vertientes de nutrición, tecnología alimentaria, seguridad alimentaria y gastronomía), d) Desarrollo de un formulario en soporte informático, con la ficha de datos antropométricos y encuestas anteriores, para ser rellenado mediante la utilización de tablets o móviles en el propio IES, e) Proceso de traspaso de los datos e información obtenida a una hoja de Excel, f) Tratamiento estadístico de resultados, g) Elaboración de una memoria final (en formato word y power point) para la discusión y exposición de resultados en el aula.

A su vez, una alumna se encargó de actuar de coordinadora de todas y cada una de las actividades anteriores y otro alumno actuó como notario, de manera ajena e imparcial al proceso, registrando cada una de las incidencias y medidas correctoras que iban ocurriendo. El resto del alumnado que no actuó como coordinador o responsable, fue encuadrado como participante en cualquiera de las actividades desarrolladas que se han nombrado en el párrafo anterior.

\subsection{Encuestas de conocimientos alimentarios}


El estudio del presente proyecto incluye a todos los alumnos de Ciclos Formativos del IES Fuensanta. Como ya se ha comentado, la encuesta de conocimientos nutricionales abarcó las áreas de nutrición, tecnología alimentaria, seguridad alimentaria y gastronomía. Incluyó una serie de preguntas con cuatro opciones de respuesta de las que sólo una era correcta. Esta encuesta fue realizada todos los lunes del 6 al 27 de noviembre en horario de mañana y los miércoles 15 y 22 de noviembre en horario de tarde, a fin de incluir a todos los alumnos que asisten al IES Fuensanta, tanto en horario de mañana como de tarde. El número de alumnos encuestados así como los ciclos a los que pertenecían aparecen en la siguiente tabla

Tabla 1.

Ciclos Formativos con número de alumnos sobre los que se realizó la encuesta.

\begin{tabular}{cccc}
\hline Ciclo formativo & $\begin{array}{c}\mathbf{N}^{\mathbf{0}} \\
\text { alumnos }\end{array}$ & Ciclo formativo & $\begin{array}{c}\mathbf{N}^{\mathbf{0}} \\
\text { alumnos }\end{array}$ \\
\hline $\begin{array}{c}\text { Dietética } \\
\text { Cuidados auxiliares de } \\
\text { enfermería }\end{array}$ & 25 & $\begin{array}{c}\text { Anatomía Patológica y } \\
\text { Citodiagnóstico }\end{array}$ & 37 \\
Higiene Bucodental & 35 & Instalación y amueblado & 9 \\
Laboratorio clínico & 40 & Sistemas de Telecomunicaciones & 30 \\
Emergencias sanitarias & 15 & $\begin{array}{c}\text { Instalaciones de } \\
\text { Telecomunicación }\end{array}$ & 32 \\
\hline
\end{tabular}

\subsection{Determinación de medidas antropométricas}

La determinación de medidas antropométricas sobre los mismos alumnos a los que se les realizó la encuesta, fue llevada a cabo por alumnado tanto de $4^{\circ}$ curso del Grado de Ciencia y Tecnología de Alimentos como de $2^{\circ}$ curso del CFGS de Dietética. El protocolo seguido para la realización de estas medidas antropométricas fue el que se sigue habitualmente en las prácticas de la asignatura Nutrición Aplicada y publicado en forma de guía (Cámara - Martos et al.2016). Las medidas antropométricas realizadas y el procedimiento seguido se indican a continuación:

a) Determinación de la altura: realizada con un tallímetro portátil (ADE MZ10017). El individuo de pie, con los talones y los pies juntos apoya la cara posterior de los glúteos y la parte superior de la espalda en la pared donde se encuentra colocado el tallímetro portatil. A continuación el evaluador, colocando las manos de la mandíbula del sujeto con los dedos tamando los procesos matoideos, desciende el vertex del tallímetro hasta el cabello del sujeto apretando lo más que se pueda. La cabeza del sujeto a medir debe colocarse en el plano de Frankfort que es aquel en el que el margen inferior de la órbita ocular está alineado horizontalmente con el trago de la oreja, o con el meato auditivo externo.

b) Determinación del perímetro medio del brazo. La circunferencia (o perímetro) del brazo se mide en el punto medio entre el olécranon y el acromión de la escápula. Para identificar este punto, el brazo no dominante (izquierdo en el caso de las personas diestras y derecho en las zurdas), debe ser flexionado $90^{\circ}$ a nivel de la articulación codo y el antebrazo se coloca con la palma de la mano hacia abajo sobre la mitad del torso. Con una cinta métrica se mide la longitud del brazo, siendo la mitad de esta longitud el punto en el que se hará la determinación, en la que se sitúa una marca con rotulador de tinta soluble en agua.

Para tomar la medida de la circunferencia del brazo, el sujeto debe dejar caído el brazo de forma relajada y sin que apoye en ningún sitio. La medición se realiza con 
cinta métrica en el lugar marcado, para lo cual la cinta ha de ajustarse firmemente pero sin que haga presión sobre el tejido adiposo.

c) Determinación de pliegues cutáneos. Se realiza para la evaluación del porcentaje de grasa de un individuo. Se considera que existe una relación constante entre la grasa subcutánea y la grasa corporal total, podremos valorar ésta, estimando la cantidad del tejido adiposo subcutáneo. Este tipo de mediciones se realizan con un caliper o plicómetro (Modelo Holtain) a $1 \mathrm{~cm}$ aproximadamente por debajo de donde estamos tomando el pliegue con los dedos, estando la musculatura relajada en el momento de la medición. Estas medidas han de realizarse por triplicado, siendo el resultado la media de las tres determinaciones. En la determinación de los pliegues cutáneos se escogieron los pliegues tricipital, bicipital, subescapular y suprailíaco.

Pliegue tricipital. El lugar para determinarlo es el mismo que elegimos para la determinación del perímetro medio del brazo. Es decir, para su medición correcta elegiremos el brazo no dominante, a menos que algún tipo de circunstancia lo impida o desaconseje. El individuo estará de pie, con el brazo desnudo, caído y relajado, o bien sentado sin que el brazo se apoye en ninguna superficie. El lugar exacto de la medición será justo en el punto medio entre el acromion de la escápula y el extremo del codo, para ello se medirá entre dichos puntos con una cinta métrica mientras el individuo mantiene el antebrazo en ángulo de $90^{\circ}$ con respecto al brazo. Una vez relajado el brazo se aprieta suavemente con los dedos pulgar e índice de la mano izquierda elevando el pliegue cutáneo y haciendo descender el paquete muscular. Para asegurarnos de no tomar masa muscular conviene que el paciente contraiga y relaje los músculos del brazo intermitentemente, sin que dejemos de aplicar los dedos suavemente.

Pliegue bicipital. Es un pliegue vertical localizado en la cara anterior del brazo a nivel del punto medio sobre el vientre del músculo bíceps. Antes de realizar la medida el brazo debe estar relajado y en posición estirada. Para realizar la medición procedemos de manera similar al pliegue tricipital. Con los dedos pulgar e índice levantamos el pliegue cutáneo con cuidado de no elevar también el paquete muscular y a continuación cogemos el pliegue con las pinzas del caliper.

Pliegue subescapular. Es un pliegue oblicuo, tomado con $45^{\circ}$ de inclinación y 1 - $2 \mathrm{~cm}$ por debajo del ángulo inferior de la escápula. Es una de las medidas necesarias para determinar el peso corporal si éste no puede ser tomado directamente. El examinador pellizca una doble capa de piel y tejido graso subcutáneo entre los dedos índice y pulgar, en una línea imaginaria que une el ángulo inferior de la escapula y el codo. El pliegue es mantenido mientras se posiciona el plicómetro perpendicularmente a la longitud del pliegue.

Pliegue suprailíaco. Pliegue oblicuo, paralelo al ángulo natural de la cresta ilíaca, tomado en la línea axilar anterior, encima de la cresta ilíaca. Localizamos el hueso de la cadera y justo por encima, a una distancia de $2.5 \mathrm{~cm}$ hacia adelante determinamos el pliegue.

d) Determinación de los perímetros de cintura y cadera. Para la determinación del perímetro de la cintura, con el abdomen relajado (después de una espiración profunda), se determina el perímetro de la circunferencia horizontal a la altura de la parte más estrecha del torso. En la determinación del perímetro cadera/glúteos, con el 
sujeto de pie y erguido, se mide el perímetro de la circunferencia horizontal máxima a la altura de los glúteos. El sujeto debe estar en ropa interior o con un bañador ligero. La persona que realiza la medición se debe colocar en cuclillas lateralmente al sujeto, a fin de ver los glúteos en toda su extensión.

e) Bioimpedancia. La utilización de la impedancia bioeléctrica como técnica para determinar el porcentaje de grasa corporal de un individuo, consiste en aplicar al cuerpo del sujeto a valorar una corriente de pequeña intensidad. Parte del hecho de que el cuerpo consta de dos componentes, una masa grasa y un compartimento magro formado por los huesos, músculos, agua y electrolitos, con capacidad para conducir la corriente eléctrica. Se utilizó una báscula de bioimpedancia (modelo TANITA BC418 MA) para la determinación del porcentaje de grasa corporal total así como en brazo izquierdo, brazo derecho, pierna izquierda, pierna derecha y abdomen; porcentaje de masa muscular; porcentaje de hidratación; metabolismo basal y determinación del peso corporal total.

\section{RESULTADOS}

El desarrollo del presente proyecto de innovación docente ha permitido a los alumnos trabajar de manera autónoma, solucionando problemas y tomando decisiones por ellos mismos con el objetivo de sacarlo adelante de manera satisfactoria. Dicho empeño, no estuvo exento de situaciones conflictivas sobre todo en los casos en los que el comienzo de una tarea dependía de la finalización de otra y así como en aquellos casos en los que se producían malentendidos por la aplicación whatsapp utilizada.

La actividad de realización de las encuestas y medición antropométrica en el IES Fuensanta, que podría haber presentado una mayor número de situaciones problemáticas, al tener que coordinar a muchas personas en un periodo corto de tiempo, se desarrolló con escasas incidencias. Además, esta fase del desarrollo del proyecto permitió la puesta en contacto directo entre los alumnos del Grado de Ciencia y Tecnología de los Alimentos y los de CFGS de Dietética estableciéndose una serie de vínculos profesionales bastante positivos.

Los datos obtenidos en el IES Fuensanta fueron pasados a una hoja de Excel, analizados estadísticamente mediante el programa SPSS versión 15.0 y presentados en la clase de Nutrición Aplicada durante el día 18 de diciembre de 2018. Aunque la información recopilada permitirá la realización de un estudio mucho más exhaustivo por parte del profesorado que se publicará en revistas especializadas de docencia y/o investigación, el informe presentado por el alumnado muestra que en relación a las encuestas alimentarias realizadas, el conocimiento de los alumnos en gastronomía es mayor que los conocimientos en nutrición, seguridad y tecnología alimentaria. Estos resultados son coherentes si se tiene en cuenta que la mayor parte de los alumnos encuestados no cursan estudios relacionados con estas materias, y el mayor conocimiento en gastronomía puede proceder de su cultura alimentaria o del ámbito familiar.

El mayor desconocimiento se produjo en las preguntas relacionadas con la seguridad alimentaria, si bien en el caso de los alumnos que cursan estudios relacionados con las ciencias de la salud, el porcentaje de alumnos suspensos (con nota inferior a 5) alcanzó el $70 \%$ frente a los alumnos de ciclos formativos de otras áreas, con un $90 \%$ de suspensos.

A partir del Índice de Masa Corporal (IMC) o relación entre el peso $(\mathrm{Kg})$ y la talla al cuadrado (m2), se observó que tan solo un 30\% de los alumnos encuestados se encontraba en situación de normopeso, frente a un $50 \%$ que presentaron sobrepeso u obesidad. El $20 \%$ de los alumnos valorados presentaron un IMC inferior 
al normal. De acuerdo a lo anterior, un porcentaje bastante elevado de alumnos presentaron altos porcentajes de grasa corporal (medida tanto por bioimpedacia como por pliegues cutáneos) y de relación cintura/cadera muy superiores a los recomendados para su edad. Así mismo, el informe realizado por el alumnado no observa ninguna correlación estadística entre puntuación obtenida en la encuesta alimentaria y su IMC, si bien este aspecto merecerá un tratamiento estadístico mucho más exhaustivo por parte del profesorado que ha coordinado el proyecto.

\section{CONCLUSIONES}

Con el proyecto que hemos desarrollado se ha introducido al alumnado a en situaciones reales que de una manera inminente va a comenzar a desarrollar nada más finalizar sus estudios y puedan consigan acceder al mercado laboral. Esta es una de las principales razones por las que el proyecto se realiza con estudiantes de último curso del grado universitario. Los alumnos han tomado de manera autónoma sus propias decisiones con la obtención de resultados bastante satisfactorios, han sabido resolver los problemas que se les ha ido planteando y han puesto en práctica los conocimientos teóricos que han adquirido durante su formación universitaria. Finalmente han podido comprobar, mediante la realización del presente trabajo de campo, la importancia de tener una buena educación nutricional en el mantenimiento de un buen estado de salud.

Como líneas futuras de mejora, considerando los elevados porcentajes obtenidos, de alumnado en situación de sobrepeso y obesidad, así como la escasa información y conocimiento, que la población estudiada tiene en general sobre temas de nutrición y seguridad alimentaria, se propone para futuras repeticiones del proyecto en los próximos cursos académicos, la inclusión final de una o varias charlas informativas. Dichas charlas se realizarían en el mismo centro donde se ha desarrollado la actividad y serían expuestas por los alumnos. Se trataría de que ellos mismos les informen y muestren a sus propios compañeros las enormes repercusiones negativas que tienen para la salud, el sobrepeso y la obesidad, así como la importancia de estar bien informado sobre temas de nutrición y seguridad alimentaria como base esencial para una vida plena y saludable.

\section{Agradecimientos}

Este proyecto ha sido financiado por la Universidad de Córdoba en su convocatoria de Proyectos de Innovación y Buenas Prácticas Docentes. Modalidad 4. Proyectos que impulsen la colaboración con centros docentes públicos no universitarios. Curso $2017 / 2018$.

\section{REFERENCIAS}

Cámara-Martos, F., Moreno-Rojas, R. \& Moreno-Ortega, A. (2016). Protocolo de Prácticas de Antropometría. Córdoba: Ediciones Don Folio.

LOE. Ley Orgánica 2/2006, de 3 de mayo, de Educación (BOE de 4 de mayo).

LOGSE. Ley Orgánica General del Sistema Educativo de 2 de octubre de 1990 (BOE de 4 de octubre). 